\title{
Propriété intellectuelle et université
}

\section{Entre la libre circulation des idées et la privatisation des savoirs}

\author{
Marc Couture \\ Marcel Dubé \\ Pierrick Malissard
}

Accès aux chapitres individuels du manuscrit final de l'ouvrage
publié en 2010 aux Presses de l'Université du Québec

Informations sur l'ouvrage imprimé, dans le site des PUQ : http://puq.ca/catalogue/collections/propriete-intellectuelle-universite-3699.html

Cliquez sur un titre pour accéder au chapitre en format PDF.

Introduction

Chapitre 1 La propriété intellectuelle : nature et portée juridique

Chapitre 2 Les formes de la propriété intellectuelle et leurs domaines d'application

Chapitre 3 La titularité de la propriété intellectuelle

Chapitre 4 La protection et l'exploitation de la propriété intellectuelle

Chapitre 5 La propriété intellectuelle : origine et évolution

Chapitre 6 L'université et la commercialisation des innovations techniques

Chapitre 7 La diffusion et le partage de l'information scientifique

Chapitre 8 L'enseignement médiatisé et le droit d'auteur

Chapitre 9 Les étudiants et la propriété intellectuelle

Chapitre 10 La réglementation de la propriété intellectuelle dans les universités canadiennes

Conclusion, annexes et références 